\title{
Influence of Sex Differences on Temporal Sequence of Sensations after Ingesting Fruit-Flavored Tea-A Preliminary Study
}

\author{
Eimi Sato', Emi Tamagawa1, Kumi Naito', Mao Nirasawa1, Ichiro Ashida ${ }^{1}$, Satomi Miyaoka ${ }^{2}$, \\ Yozo Miyaoka ${ }^{1}$ \\ ${ }^{1}$ Department of Health and Nutrition, Niigata University of Health and Welfare, Niigata, Japan \\ ${ }^{2}$ Department of Mental Health Science, Graduate School of Rehabilitation, Niigata University of Rehabilitation, Murakami, \\ Japan \\ Email:miyaoka@nuhw.ac.jp
}

How to cite this paper: Sato, E., Tamagawa, E., Naito, K., Nirasawa, M., Ashida, I., Miyaoka, S. and Miyaoka, Y. (2018) Influence of Sex Differences on Temporal Sequence of Sensations after Ingesting Fruit-Flavored Tea-A Preliminary Study. Food and Nutrition Sciences, 9, 676-682. https://doi.org/10.4236/fns.2018.96051

Received: May 16, 2018

Accepted: June 16, 2018

Published: June 19, 2018

Copyright (c) 2018 by authors and Scientific Research Publishing Inc. This work is licensed under the Creative Commons Attribution International License (CC BY 4.0).

http://creativecommons.org/licenses/by/4.0/

\section{cc) (i) Open Access}

\begin{abstract}
The goal of this preliminary study was to examine the effect of participants' sexes on the temporal sequence of dominant sensations elicited by fruit-flavored teas. Twelve healthy young adults were assigned to male $(n=6)$ and female $(n=6)$ groups. Both groups were evaluated for four sensations using four fruit-flavored teas with $0.05 \mathrm{M}$ sucrose using the temporal dominance of sensations (TDS) method. Only two sensations, sweetness and fruitiness, were consistently reported in the two groups. The male group first reported fruitiness and then sweetness as the dominant sensation following ingestion of the samples. Conversely, the female group reported these two dominant sensations in the reverse order. Significant dominant durations between the two sensations largely varied among the samples in both groups. These results suggest that there are sex-based differences in the temporal sequence of dominant sensations elicited by fruit-flavored teas as evaluated by the TDS method.
\end{abstract}

\section{Keywords}

Flavor, Tea, Sweetness, Fruitiness, Temporal Dominance of Sensations, Sex Differences

\section{Introduction}

Investigation of interactions between taste and smell is a keystone for understanding of flavor perception, since the term "flavor" as a sensation resulting from interactions of taste, smell (aroma), and trigeminal sensations [1] [2]. The intensity of sweetness can be enhanced by adding some odorants to sweet solu- 
tions [3] [4]. Previous studies have evaluated this enhancement using magnitude estimation methods and have paid little attention to temporal aspects of the enhancement. Recently, temporal dominance of sensations (TDS) method has been used to understand temporal sequence of the perception of sensory attributes of drinks and foods [5]. Di Monaco and colleagues examined the temporal aspects of the enhancement of six sensations, including sweetness and fruitiness (strawberry flavor) in four model beverages using TDS [6]. On the other hand, concentrations of the used samples were much higher (up to $1.2 \mathrm{M}$ in [3] and 0.56 $M$ in [4]) than those in everyday beverages. Actually, a survey analyzed the saccharides in 42 sweet snacks and beverages in Japan and reported that three sweet teas contained an average of $0.05 \mathrm{M}$ to $0.18 \mathrm{M}$ sucrose [7]. Although the sex of study participants considerably affects various sensory functions [8], no previous studies have considered the participants' sex when examining temporal aspects of these flavor enhancements [5] [9] [10] [11]. In this study, we examined the effect of participants' sex on temporal sequence of flavor sensations elicited by fruit-flavored teas with an average level of sugar $(0.05 \mathrm{M})$ preliminarily.

\section{Materials and Methods}

\subsection{Participants}

Twelve healthy young adults ( 6 males and 6 females, $20.7 \pm 1.2$ years old) participated in this study after providing informed consent. The participants were assigned to male and female groups according to their sex. All of the participants had no subjective problems in gustatory and olfactory functions and passed both gustatory (Taste Test Kit-PRO, Delico co., Ltd., Osaka, Japan) and olfactory (Five Standard Odors for selection of panel members set of center concentrations, Daiichi Yakuhin Sangyo Co., Ltd., Tokyo, Japan) acuity tests. The Ethics Committee of Niigata University of Health and Welfare approved the experiments (\#17859-170711).

\subsection{Samples}

Based on previous studies [3] [4], four fruit-flavored teas were used as samples in this study. The fruit-flavored teas were prepared by adding commercially available lemon, strawberry, peach, and vanilla flavors to non-flavored tea $(\mathrm{Mu}-$ tou koucha (Non-sugar tea), Kirin Beverage Company, Limited, Tokyo, Japan). Sucrose at a concentration of $0.05 \mathrm{M}$ was added to all four samples. Three concentrations $(0.5,1.5$, and $3.0 \mu \mathrm{l} / \mathrm{ml})$ of the flavors were added to the tea and it was served at around $31^{\circ} \mathrm{C}$ to the participants. Only data collected from the highest concentration $(3.0 \mu \mathrm{l} / \mathrm{ml})$ were analyzed in the present study as this concentration yielded the most consistent results.

\subsection{Recording Tools}

A TDS system (J-SEMS TDS\&TI, MediaEye, Tokyo, Japan) was used for recording and analyzing sensory evaluation data, and four items that elicit four differ- 
ent sensations, i.e., sweetness, sourness, astringency, and fruitiness, were utilized for sensory evaluation. The sensation of "flavorlessness" was also utilized to clarify the situation that the participant selected none of the four items. The recording system consisted of a base unit and four cordless extension units of tablet terminators, so maximum four participants could use the system at the same time.

\subsection{Procedures}

After training participants on the TDS system, each participant was instructed to properly rinse their mouth with tap water. For practice purposes, they were presented a non-flavored sample $(10 \mathrm{ml}$ ). This was followed by 12 (four flavors $\times$ three concentrations) flavored samples (10 $\mathrm{ml}$ each). The 12 samples were presented in a random order, but each flavor was presented in an increasing concentration from low $(0.5 \mu \mathrm{l} / \mathrm{ml})$ to high $(1.5 \mu \mathrm{l} / \mathrm{ml}$ and $3.0 \mu \mathrm{l} / \mathrm{ml})$. The participants were instructed to drink, taste, and swallow each sample and to press one of the five buttons for evaluation items on a tablet device that recorded their responses. The time of swallowing was measured from the moment of ingestion. An interval of $30 \mathrm{~s}$ was set from the start to the end of each evaluation, and the participants were instructed to rinse their mouths with tap water at the end of each trial. A one-minute interval was allowed between trials. Participants were asked not to discuss their evaluations with each other.

\subsection{Data and Statistical Analyses}

Comma separated values (CSV) formatted data were obtained from the TDS system and the CSV data were processed using the " $R$ " programming language to calculate basic statistics, such as averages and standard deviations [12]. Two sensations, sweetness and fruitiness, were subjected to the following analysis, since only these two sensations were consistently reported at significant levels in both the groups and across the four flavored samples. First, the TDS curves of the male and female groups were obtained by the following two steps: i.e., 1) calculation of the average dominance values at each time point by $d_{\mathrm{i}}=\Sigma s_{\mathrm{ij}} / n$, where $d_{\mathrm{i}}$ is an averaged dominance at $\mathrm{i}^{\text {th }}$ second $(\mathrm{i}=0,1, \cdots, 30), s_{\mathrm{ij}}$ is a response of $\mathrm{j}^{\text {th }}$ participant $(\mathrm{j}=1,2, \cdots, n)$ at $\mathrm{i}^{\text {th }}$ second, and $n$ is the number of participants in the male or female group, and 2) smoothing of the averaged dominance points using the spline interpolation [13]. Second, differences in the dominance ratios between the two sensations were calculated in the same sample. Third, the differences in dominance ratios were compared based on two binomial proportions [14]. Only significant differences were plotted on a scatter diagram, which we called a TDS difference curve (TDS-DC). Finally, the first time-points at which the two sensations significantly differed were compared between the male and female groups for the individual samples.

\section{Results and Discussion}

Only two, sweetness and fruitiness, of the five sensations evaluated in this study 
showed consistent and statistically significant responses to the used samples. Figure 1 presents average TDS curves of these two sensations reported from the beginning of sample ingestion to the end of the $30 \mathrm{~s}$-duration for the evaluation of sensations in the male and female groups. The sensation of fruitiness appeared first and was followed by that of sweetness in the male group (Figure 1(a)). This order of appearance was reversed in the female group (Figure 1(b)). A t-test showed that the average beginning time of fruitiness in the male group across the four samples was significantly earlier than in the female group $(P<$ 0.005). The t-test also showed that the average duration of the sensation of fruitiness in the male group was significantly shorter than in the female group $(P<$ 0.05). Since the male group did not reach a significant level of sensation for sweetness in the lemon-flavored sample (Figure 1(a)), we were unable to perform statistical examinations on this sensation.

Figure 2 depicts TDS-DC curves for the four samples in the male and female groups. There were significant differences in the duration of the fruitiness and sweetness sensations between the two groups. The TDS-DC curves revealed that fruitiness was more dominant than sweetness in all four samples and in both

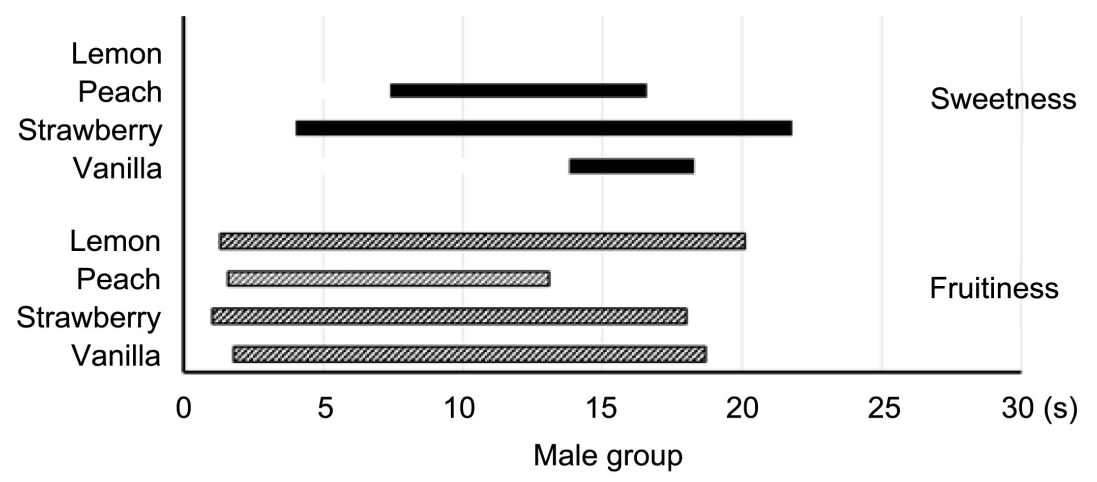

(a)

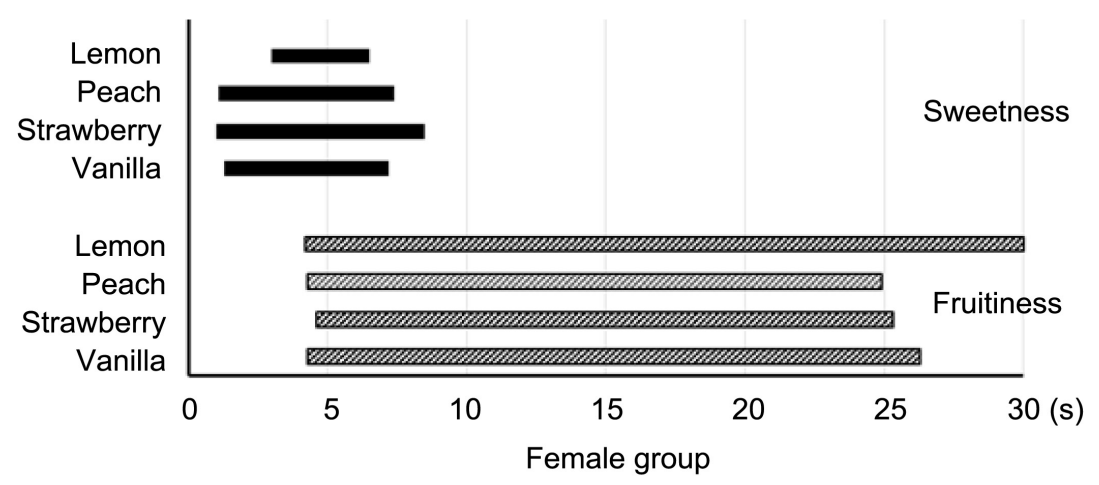

(b)

Figure 1. Average significant durations of TDS curves. The beginning, duration, and end times for statistically significant levels of sweetness and fruitiness in four fruit-flavored tea samples reported by the male (a) and female (b) groups. Lemon: lemon-flavored tea, Peach: peach-flavored tea, Strawberry: strawberry-flavored tea, Vanilla: vanilla-flavored tea. Closed bars indicate "sweetness" and hatched bars indicate "fruitiness". 


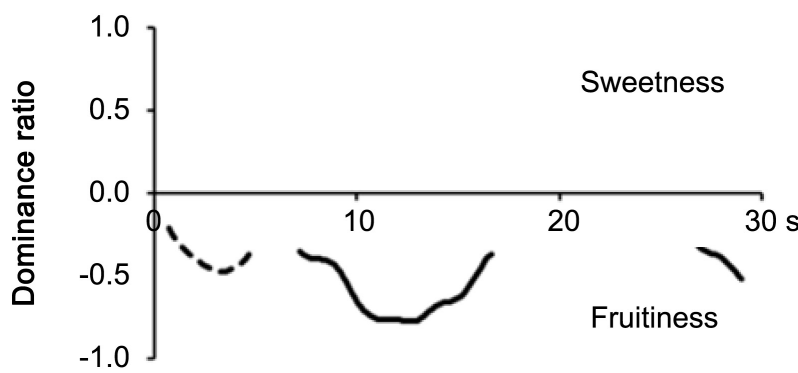

Lemon

(a)

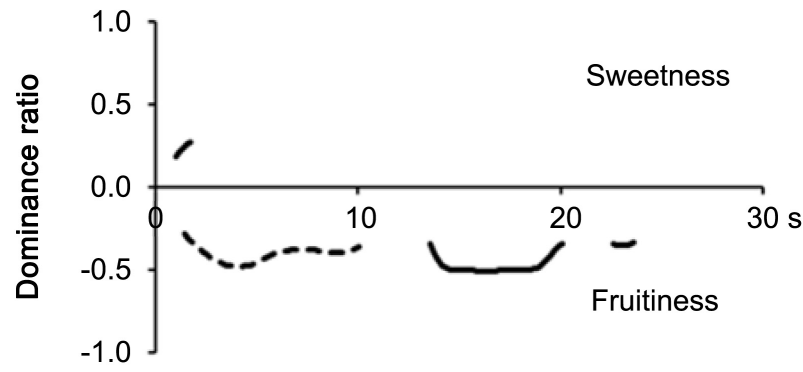

Strawberry

(c)

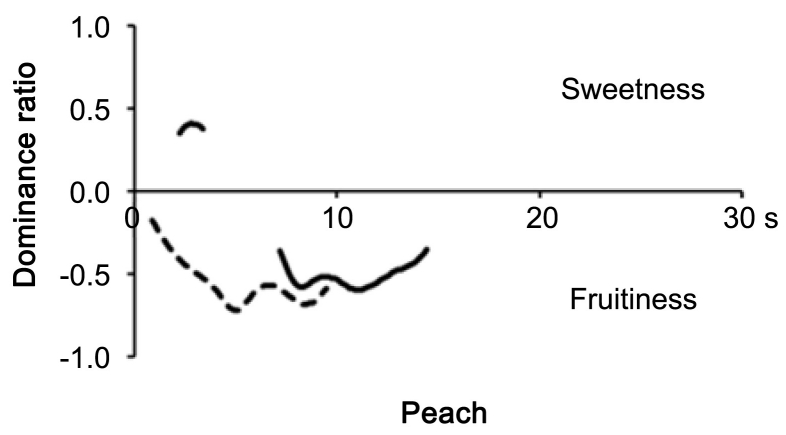

(b)

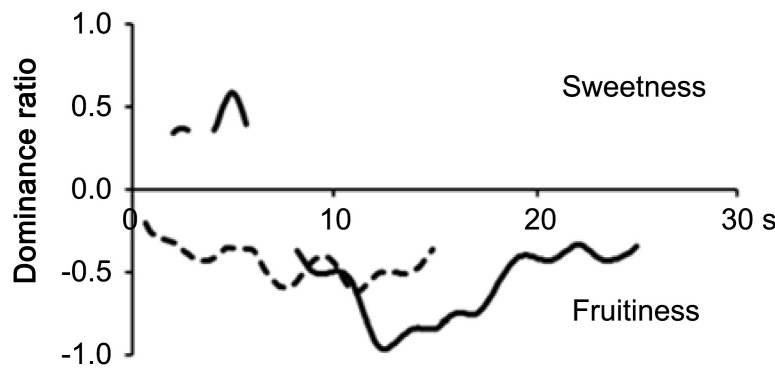

Vanilla

(d)

Figure 2. TDS difference curves for the four samples (a)-(d) in the male and female groups. TDS difference curves for the lemon-flavored tea (Lemon; (a)), for the peach-flavored tea (Peach; (b)), for the strawberry-flavored tea (Strawberry; (c)), and for the vanilla-flavored tea (Vanilla; (d)). Broken lines indicate the male group and solid lines indicate female group. Positive values on the vertical axis imply "sweetness" dominance, while negative values imply "fruitiness" dominance. Only significant differences were plotted on the TDS difference curves.

groups. The basic features of sweetness and fruitiness in the TDS-DC curves were consistent among the samples, but there were some differences between them. For example, both the maximum dominance ratio (around -0.5) and the dominant duration (6.7 s) of the strawberry-flavored tea in the female group (Figure 2(c)) were less than half of the vanilla-flavored tea (close to -1.0 and 17 s; Figure 2(d)). However, any statistical examinations were not applicable for the TDS-DC curves, since there were only two curves without variation of data by twice experimental repetitions in the present study.

A previous study [5] using the TDS method examined temporal changes in six sensations, including sweetness [by sucrose, $40 \mathrm{~g} / \mathrm{l}(0.12 \mathrm{M})$ ] and fruitiness (by strawberry flavor, $50 \mu \mathrm{l} / \mathrm{l}$ ), for $100 \mathrm{~s}$ after ingestion of the sample. This study demonstrated that only sweetness and strawberry flavor reached statistically significant levels at the beginning of the ingestion [10], just like the present study (Figure 1). However, the duration of the sensations was about $60 \mathrm{~s}$ for sweetness and $30 \mathrm{~s}$ for strawberry flavor with a brief break [Figure 3 in [5]]. These results are inconsistent with the ones obtained in the present study, in which the fruitiness sensation lasted much longer than sweetness in both the groups (Figure 1). Large differences in the concentrations of sucrose $(0.12 \mathrm{M}$ vs. $0.05 \mathrm{M})$ and 
strawberry flavor $(50 \mu \mathrm{l} / \mathrm{l}$ vs. $3.0 \mathrm{ml} / \mathrm{l})$ between these studies are likely to be responsible for this inconsistency. It is also worth noting that the study by Di Monaco and colleagues [5] used a mixed group consisting of male and female participants, whereas the present study divided the participants into male and female groups. This study also compared the times at which the two sensations first reached significant levels and the durations for which the sensations sustained between the two groups. As a result, the findings of this study strongly suggest that sex differences affect temporal sequence of flavor perception of the tea samples (Figure 1 and Figure 2).

A previous study [10] suggested that more than 16 participants were needed for the experiment of twice repetition, like the present study $\left(n_{0}>5 /\left(p_{0}\left(1-p_{0}\right)\right.\right.$ $r$, where $n_{0}$ is minimum number of participants, $p_{0}$ is inverse of number of sensations including flavorlessness, and $r$ is number of repetition). Although the small number of participants (12 in the two groups) is the first and biggest limitation of this study, the differences in temporal sequence shown between the male and female groups in this study (Figure 1 and Figure 2) are clear and might be unaffected by increasing the number of participants. In the near future, however, it is necessary to confirm actually the present finding by experiments using more participants. The mechanism for the present finding that sex-based differences in the temporal sequence of dominant sensations elicited by fruit-flavored teas remains unclear, which is the second limitation of this study. Sex hormones, like oestrogen, may be related to the finding [8] [15]. To examine the relation, using postmenopausal, instead of the young, women as participants is likely to be valid. Besides, it is also necessary to clarify the effect of basic sucrose concentration on the finding, since there is a wide distance between previous (e.g., $0.56 \mathrm{M}$, [4]) and present $(0.05 \mathrm{M})$ studies in the concentration.

\section{Conclusion}

In conclusion, two sensations, sweetness and fruitiness, dominantly appeared after ingesting four fruit-flavored teas (lemon, peach, strawberry, and vanilla) by a TDS method in both male and female groups. The male group perceived fruitiness first and sweetness second, while the female group reported in the reverse order.

\section{Acknowledgements}

This study was partially supported by Grants-in-Aid for Scientific Research from the Ministry of Education, Science and Culture of Japan (No. 26350106 to SY and No. 16K00830 to YM).

\section{References}

[1] Prescott, J. (1999) Flavour as a Psychological Construct: Implications for Perceiving and Measuring the Sensory Qualities of Foods. Food Quality and Preference, 10, 349-356. https://doi.org/10.1016/S0950-3293(98)00048-2 
[2] Small, D.M. and Prescott, J. (2005) Odor/Taste Integration and the Perception of Flavor. Experimental Brain Research, 166, 345-357. https://doi.org/10.1007/s00221-005-2376-9

[3] Frank, R.A. and Byram, J. (1988) Taste-Smell Interactions Are Tastant and Odorant Dependent. Chemical Senses, 13, 445-455. https://doi.org/10.1093/chemse/13.3.445

[4] Green, B.G. Nachtigal, D. Hammond, S. and Lim, J. (2012) Enhancement of Retronasal Odors by Taste. Chemical Senses, 37, 77-86. https://doi.org/10.1093/chemse/bjr068

[5] Di Monaco, R., Miele, N.A., Volpe, S., Picone, D. and Cavella, S. (2014) Temporal Sweetness Profile of MNEI and Comparison with Commercial Sweeteners. Journal of Sensory Studies, 29, 385-394. https://doi.org/10.1111/joss.12119

[6] Di Monaco, R., Su, C., Paolo, M. and Cavella, S. (2014) Temporal Dominance of Sensations: A Review. Trends in Food Science \& Technology, 38, 104-112. https://doi.org/10.1016/j.tifs.2014.04.007

[7] Yamamoto, S., Taniguchi, H., Sarukura, N., Tsao, H., Tseng, A. and Takeichi, H.(2009) Development of a Food Composition Database of Mosaccharides and Disaccharides in Sweet Snacks and Beverages (In Japanese with English Abstract). Journal of the Japan Dietetic Association, 52, 22-25.

[8] Velle, W. (1987) Sex Differences in Sensory Functions. Perspectives in Biology and Medicine, 30, 490-522. https://doi.org/10.1353/pbm.1987.0015

[9] Labbe, D., Schlich, P., Pineau, N., Gilbert, F. and Martin, N. (2009) Temporal Dominance of Sensations and Sensory Profiling: A Comparative Study. Food Quality and Preference, 20, 216-221. https://doi.org/10.1016/j.foodqual.2008.10.001

[10] Pineau, N., Schlich, P., Cordelle, S., Mathonniere, C., Issanchou, S., Imbert, A., Rogeaux, M., Etievant, P. and Koster, E. (2009) Temporal Dominance of Sensations: Construction of the TDS Curves and Comparison with Time-Intensity. Food Quality and Preference, 20, 450-455. https://doi.org/10.1016/j.foodqual.2009.04.005

[11] Barron, D., Pineau, N., Matthey-Doret, W., Ali, S., Sudre, J., Germain, J.C., et al. (2012) Impact of Crema on the Aroma Release and the In-Mouth Sensory Perception of Espresso Coffee. Food \& Function, 3, 923-930. https://doi.org/10.1039/c2fo30046j

[12] Ihaka, R. and Gentleman, R. (1996) R: A Language for Data Analysis and Graphics. Journal of Computational and Graphical Statistics, 5, 299-314.

[13] Crawley, M.J. (2002) Statistical Computing: An Introduction to Data Analysis using S-Plus. Wiley, UK.

[14] Kiemele, M.J. (1997) Basic Statistics: Tools for Continuous Improvement. Air Academy Press, Colorado Springs, USA.

[15] Diamond, J., Dalton, P., Doolittle, N. and Breslin, P.A.S. (2005) Gender-Specific Olfactory Sensitization: Hormonal and Cognitive Influences. Chemical Senses, 30, i224-i225. https://doi.org/10.1093/chemse/bjh195 